\title{
EDITORIAL
}

\section{THE LONG AND WINDING ROAD}

In reality, the road to a marketed drug is often far from the frequently depicted linear path. Extracting practical insights and lessons from such experiences by discussing drug discovery

'Case histories' is the aim of an inaugural forum this month.

1in 5,000. For many of our readers, this statistic will be familiar as the estimated fraction of compounds entering preclinical testing that eventually become marketed drugs. So, among the multitude of uncertainties associated with the drug discovery process, one thing is for sure: there is something quite special about any compound that makes it over the many hurdles on the way to the market.

It was with this thought in mind that we established our series of 'Case history' articles, each of which looks at the story behind the discovery and development of a particular drug or drug class. Written by people who were closely involved in the process - who can therefore tell the reader exactly what it was like - the aim of these articles is to both provide inspiration for future drug discoverers, and to draw out the key challenges overcome and decisions made that ultimately resulted in a marketed therapeutic, rather than one of the vast number of other drug candidates that fall by the wayside.

Extending this idea, this month, in collaboration with Nature Biotechnology and IBC, Nature Reviews Drug Discovery will be holding a 'Case Histories' forum in Boston (for more information, please see www.ibclifesciences.com/casehistories). The forum will involve interactive discussions of the stories behind five recently approved pioneering drugs, each led by an expert in the field.

The drugs selected for analysis have been chosen not just for their innovative nature, but also for their potential to illustrate issues of wider relevance to drug discovery in general. First to be discussed will be the kinase inhibitor imatinib (Gleevec), first approved for the treatment of chronic myelogenous leukaemia in 2001. A pioneering example of a molecularly targeted agent, the considerable clinical success of imatinib provided a major boost to what is now the most active area in anticancer drug development. Furthermore, ongoing experience with imatinib since its approval is highlighting key issues for the future of molecularly targeted agents in general, such as the origins of resistance to these agents.
Staying with the theme of molecularly targeted agents, also under discussion will be the anti-VEGF antibody bevacizumab (Avastin), whose development illustrates the perseverance that often seems to be the key to translating an innovative therapeutic approach into a marketed drug. Its approval last year for colorectal cancer could be viewed as validation of the hypothesis first put forward more than 30 years ago that targeting tumour angiogenesis would be a viable anticancer strategy.

Serendipity has also played a part in a number of drug discovery successes, although as Louis Pasteur noted, in the field of observation, chance favours only the prepared mind. The third agent discussed, the anti-HIV drug enfuvirtide (Fuzeon) - originally tested in experiments aimed at evaluating strategies in vaccine development, but ultimately becoming the first in a new class of drugs that inhibit HIV entry into host cells - is one such case. In addition, it provides an excellent example of translation of an academic research discovery to the clinic.

Although much can be learned from successes in drug development, it is also important to analyse situations in which something goes awry, as has turned out to be the case for the other two case histories to be discussed. The antibody natalizumab (Tysabri), which inhibits the migration of immune cells into the central nervous system, was given fast-track approval for multiple sclerosis last year after impressive results in clinical trials. However, its recent voluntary market withdrawal owing to deaths thought to be related to a virus that attacks the central nervous system has raised important general questions about the risk/benefit profile of this and other therapies in development that suppress aspects of the immune response. Striking the balance between benefit and risk is also a major issue raised by the last of the case histories: the story of the COX2 inhibitors. Given the impact that the withdrawal of rofecoxib (Vioxx) last year has had on the industry as a whole, philosopher George Santayana's dictum on the importance of understanding the past has particular resonance: "Those who cannot learn from history are doomed to repeat it". 